\title{
Active Vibration Distribution through Restricted Topology and Single Node Control
}

\author{
Jiqiang Wang \\ Jiangsu Province Key Laboratory of Aerospace Power Systems, Nanjing University of Aeronautics and Astronautics, \\ 29 Yudao Street, Nanjing 210016, China \\ Correspondence should be addressed to Jiqiang Wang; jiqiang_wang@hotmail.com
}

Received 10 July 2020; Revised 3 October 2020; Accepted 9 October 2020; Published 23 October 2020

Academic Editor: W. Zhang

Copyright (c) 2020 Jiqiang Wang. This is an open access article distributed under the Creative Commons Attribution License, which permits unrestricted use, distribution, and reproduction in any medium, provided the original work is properly cited.

Vibration distribution comprises both vibration control and harvesting. Active vibration distribution control represents a generic yet challenging problem to be addressed in many engineering applications. Current norm-optimization-based methods would require significant modification for its solutions. This paper proposes a restricted control method where a variety of results concerning active vibration distribution through restricted topology control and restricted single node control are obtained. Important issues associated with both parametric constraints and performance limit are also given considerations. A systematic design methodology is thus established for active vibration distribution through restricted controls. The theoretical results are exemplified with numerical examples providing detailed design procedures.

\section{Introduction}

Distribution of vibration over structural systems has been prominent in many engineering problems. In vehicles, vibration propagation is closely monitored for driving safety and passenger comfort [1]; in gas turbine and aerospace applications, vibration distribution properties are utilized for engine/aircraft health management with improved operational decision-making $[2,3]$; in power systems, transformer tank vibration is used to detect DC bias [4] and winding deformations [5]. Since vibration distribution reveals the properties of the underlying structures, it has aroused theoretical interests for feature detection and extraction. However, previous investigations have focused exclusively on system analysis (refer to [6-9] for example) and estimation [10]. The problem of active control of vibration distribution has not been explored in the literature. However, active vibration distribution control is necessitated in practical engineering. For example, vibration distribution is captured to probe the acoustic signature of naval ships and submarines, and consequently there exists a great demand for "manipulating" the distribution of vibrations for military purposes.
Different from conventional understanding of vibration distribution, a typical scenario is assumed where one region or location of vibration is suppressed while the vibration is to be enhanced over the other region or location across the structure. It is readily recognized that active vibration distribution in such a scenario actually comprises two very important areas of vibration control [11-14] and vibration energy harvesting [15-18]. Active vibration distribution thus represents a generic problem of intentionally manipulating the distribution of vibration across structures in a prescribed manner. To achieve this objective, conventional control design approaches such as $(H 2 / H \infty)$ control can indeed be utilized $[19,20]$. However, these approaches would usually require large amount of distributed sensors and actuators, while engineering experience calls upon very restricted control approaches with limited feedback capability and parameter constraints. A novel method is devised in this paper to tackle this challenging problem of active vibration distribution design using restricted control. In specific, the contributions are summarized as follows:

(1) The concept of active vibration distribution encompassing both vibration control and energy harvesting is proposed 
(2) The generic problem of active vibration distribution is tackled with restricted control

(3) Control restrictions including restricted topology, restricted single node, and restricted parameter constraints are handled

(4) Fundamental performance limit is also given considerations

The paper is structured as follows. Section 2 formulates the problem of restricted active control of vibration distribution. The solutions are given in Section 3, while Section 4 develops a novel solution of restricted active single node control. Constrained and robustness issues are considered in Section 5. Section 6 considers the problem of performance design limit. Numerical examples are given in Section 7 where detailed design procedures are illustrated with simulations. Finally, conclusions are given in Section 8 .

\section{Problem Formulation}

The problem of vibration distribution will be formulated with respect to a general $N$-nodes and unity inner coupling heterogeneous system with a networked structure subject to exogenous disturbances:

$$
\dot{x}_{i}(t)=a_{i} x_{i}(t)+\sum_{\substack{j=1 \\ j \neq i}}^{N} s_{i j} x_{j}(t)-s_{i 0} d_{i}(t), \quad i \in[1, \ldots, N],
$$

where $x_{i}(t)$ is the state variable and $a_{i}$ is the node dynamics, $s_{i j}$ is the topological interaction among the nodes, and $s_{i 0}$ is the interaction strength with the exogenous signal $d_{i}(t)$. It remains to see that representation (1) can include the general multiple DOF mass-damper-spring systems as well as the structural systems modeled by distributed agents $[21,22]$.

Other than using conventional dynamics feedback control, it is assumed that control is restricted solely to topology feedback:

$$
\begin{aligned}
& \dot{x}_{i}(t)=a_{i} x_{i}(t)+\sum_{\substack{j=1 \\
j \neq i}}^{N} s_{i j} x_{j}(t)-s_{i 0} d_{i}(t)+u_{i}(t), \quad i \in[1, \ldots, N], \\
& u_{i}(t)=\sum_{\substack{j=1 \\
j \neq i}}^{N} g_{i j} x_{j}(t),
\end{aligned}
$$

where $u_{i}(t)$ represents the control action with $g_{i j}$ being the to-be-designed topological gains. Thus restricted control implies that only topology feedback is available, yet gradually further restrictions on parameter constraints and system constraints will also be enforced. Restricted control acquires its meaning complying with engineering practice where the numbers of sensors, actuators, physical parameters, and system dynamics are all restricted.

The closed-loop system with topology feedback is

$$
\begin{aligned}
\dot{x}_{i}(t)= & a_{i} x_{i}(t)+\sum_{\substack{j=1 \\
j \neq i}}^{N}\left(s_{i j}+g_{i j}\right) x_{j}(t) \\
& -s_{i 0} d_{i}(t), \quad i \in[1, \ldots, N] .
\end{aligned}
$$

Writing into frequency response representation for a frequency $\omega$ leads to

$$
\left[\begin{array}{c}
X_{1} \\
X_{2} \\
\vdots \\
X_{N}
\end{array}\right]=\left[\begin{array}{cccc}
\left(a_{1}-j \omega\right) & s_{12}+g_{12} & \cdots & s_{1 N}+g_{1 N} \\
s_{21}+g_{21} & \left(a_{2}-j \omega\right) & \cdots & s_{2 N}+g_{2 N} \\
\vdots & \vdots & \ddots & \vdots \\
s_{N 1}+g_{N 1} & s_{N 2}+g_{N 2} & \cdots & \left(a_{N}-j \omega\right)
\end{array}\right]^{-1}\left[\begin{array}{c}
s_{10} D_{1} \\
s_{20} D_{2} \\
\vdots \\
s_{N 0} D_{N}
\end{array}\right]
$$

where $X_{i}(j \omega)$ and $D_{i}(j \omega)$ are the frequency response of $x_{i}(t)$ and $d_{i}(t)$, respectively, with dependence on frequency being omitted for easy reference. Now represent the disturbances with $D_{i}(j \omega)=\widehat{C}_{i} D(j \omega)$, where $\widehat{C}_{i}$ is a complex number representing the gain and phase shift over $D(j \omega)$; further, redefine $C_{i}=s_{i 0} C_{i}$; then (4) can be written as follows:

$$
\left[\begin{array}{c}
X_{1} \\
X_{2} \\
\vdots \\
X_{N}
\end{array}\right]=\left[\begin{array}{cccc}
\left(a_{1}-j \omega\right) & s_{12}+g_{12} & \cdots & s_{1 N}+g_{1 N} \\
s_{21}+g_{21} & \left(a_{2}-j \omega\right) & \cdots & s_{2 N}+g_{2 N} \\
\vdots & \vdots & \ddots & \vdots \\
s_{N 1}+g_{N 1} & s_{N 2}+g_{N 2} & \cdots & \left(a_{N}-j \omega\right)
\end{array}\right]^{-1}\left[\begin{array}{c}
C_{1} \\
C_{2} \\
\vdots \\
C_{N}
\end{array}\right] D,
$$

or

$$
X=(\Lambda+S+G)^{-1} \mathrm{CD}
$$

where $X=\left[\begin{array}{c}X_{1} \\ X_{2} \\ \vdots \\ X_{N}\end{array}\right]$ and $C=\left[\begin{array}{c}C_{1} \\ C_{2} \\ \vdots \\ C_{N}\end{array}\right] ; S=\left[\begin{array}{cccc}0 & s_{12} & \cdots & s_{1 N} \\ s_{21} & 0 & \cdots & s_{2 N} \\ \vdots & \vdots & \ddots & \vdots \\ s_{N 1} & s_{N 2} & \cdots & 0\end{array}\right]$

and $G=\left[\begin{array}{cccc}0 & g_{12} & \cdots & g_{1 N} \\ g_{21} & 0 & \cdots & g_{2 N} \\ \vdots & \vdots & \ddots & \vdots \\ g_{N 1} & g_{N 2} & \cdots & 0\end{array}\right]$; finally, $\Lambda=\operatorname{diag}\left(\left(a_{1}-j \omega\right)\right.$ $\left.\cdots\left(a_{N}-j \omega\right)\right)$.

Now partition (6) into two groups with attenuating variables and harvesting variables as

$$
\left[\begin{array}{c}
X_{\mathrm{va}} \\
X_{\mathrm{ve}}
\end{array}\right]=\left[\begin{array}{ll}
P_{11} & P_{12} \\
P_{21} & P_{22}
\end{array}\right]\left[\begin{array}{c}
C_{\mathrm{va}} \\
C_{\mathrm{ve}}
\end{array}\right] D
$$

where $X \equiv\left[\begin{array}{l}X_{v a} \\ X_{v e}\end{array}\right], \quad C \equiv\left[\begin{array}{l}C_{v a} \\ C_{v e}\end{array}\right], \quad$ and $\left[\begin{array}{ll}P_{11} & P_{12} \\ P_{21} & P_{22}\end{array}\right] \equiv$ $(\Lambda+S+G)^{-1}$ with appropriate dimensions determined from performance specification for $X_{1}(j \omega), X_{2}(j \omega), \ldots$, $X_{N}(j \omega)$. With no loss of generality, the first $M(M<N)$ variables $X_{\mathrm{va}}$ are required being attenuated and the remaining $X_{\mathrm{ve}}$ being harvested. The generic problem of active vibration distribution can be formulated as finding 
appropriate controls such that the following inequalities are satisfied:

$$
\begin{aligned}
& \left\|P_{11} C_{\mathrm{va}}+P_{12} C_{\mathrm{ve}}\right\|_{\mathrm{ew}}<\delta_{1}<1, \\
& \left\|P_{21} C_{\mathrm{va}}+P_{22} C_{\mathrm{ve}}\right\|_{\mathrm{ew}}>\delta_{2}>1,
\end{aligned}
$$

where the norm $\|\cdot\|_{\mathrm{ew}}$ denotes the element-wise operation; e.g., $\left\|P_{11} C_{\mathrm{va}}+P_{12} C_{\mathrm{ve}}\right\|_{\mathrm{ew}}<1$ means that $X_{1}(j \omega), \ldots$, $X_{M}(j \omega)$ are all attenuated with specification $\delta_{1}=\left[\begin{array}{lll}\delta_{1,1} & \cdots & \delta_{1, M}\end{array}\right]^{T}$. Thus, the problem of active vibration distribution through restricted topology control can be stated.

Active Vibration Distribution through Restricted Topology Control aims to find a set of restricted feedback controls (2) such that inequalities in (8) are satisfied for prescribed performance index; should the designated performance index be infeasible, explain the failure and synthesize suboptimal controllers.
It can be expected that the required design methodology must be able to determine the performance limit that has been a challenging task for any conventional design approach. These difficult problems will be handled in the following sections.

\section{Restricted Active Topology Control}

The above restricted active vibration distribution problem can be tackled by solving the inequalities in (8) for the required topological parameters $g_{i j} s$ for a specification $\left[\begin{array}{ll}\delta_{1} & \delta_{2}\end{array}\right]^{T}$. However, this can only be done by calculating matrix inversion before partition in terms of

$$
\left[\begin{array}{ll}
P_{11} & P_{12} \\
P_{21} & P_{22}
\end{array}\right] \equiv(\Lambda+S+G)^{-1} \text {. }
$$

This can be very difficult and, to relieve this problem, consider the following identity:

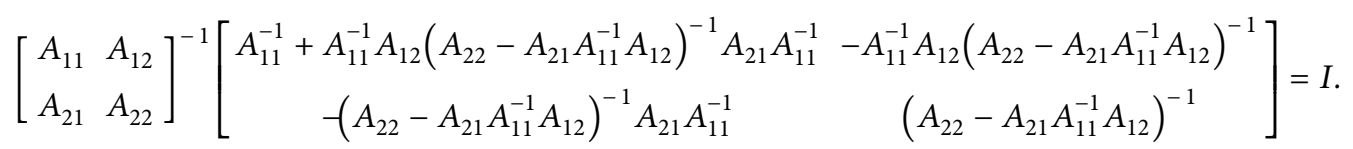

And define the partition

Then, from (9) and (10), the following can be obtained:

$$
\Lambda+S+G \equiv\left[\begin{array}{ll}
A_{11} & A_{12} \\
A_{21} & A_{22}
\end{array}\right] .
$$

$$
\left[\begin{array}{ll}
P_{11} & P_{12} \\
P_{21} & P_{22}
\end{array}\right]=\left[\begin{array}{cc}
A_{11}^{-1}+A_{11}^{-1} A_{12}\left(A_{22}-A_{21} A_{11}^{-1} A_{12}\right)^{-1} A_{21} A_{11}^{-1} & -A_{11}^{-1} A_{12}\left(A_{22}-A_{21} A_{11}^{-1} A_{12}\right)^{-1} \\
-\left(A_{22}-A_{21} A_{11}^{-1} A_{12}\right)^{-1} A_{21} A_{11}^{-1} & \left(A_{22}-A_{21} A_{11}^{-1} A_{12}\right)^{-1}
\end{array}\right]
$$

Consequently, (8) can be manipulated to be

$$
\begin{array}{r}
\left\|A_{11}^{-1}\left[C_{\mathrm{va}}-A_{12} P_{22}\left(C_{\mathrm{ve}}-A_{21} A_{11}^{-1} C_{\mathrm{va}}\right)\right]\right\|_{\mathrm{ew}}<\delta_{1}, \\
\left\|P_{22}\left(C_{\mathrm{ve}}-A_{21} A_{11}^{-1} C_{\mathrm{va}}\right)\right\|_{\text {ew }}>\delta_{2} .
\end{array}
$$

The result up to now can be summarized as follows.

Theorem 1. For a given specification $\delta_{1}=\left[\begin{array}{lll}\delta_{1,1} & \cdots & \delta_{1, M}\end{array}\right]^{T}$ and $\delta_{2}=\left[\begin{array}{lll}\delta_{2,(M+1)} & \cdots & \delta_{2, N}\end{array}\right]^{T}$, active vibration distribution through restricted topology control can be resolved by solving inequalities (13) for a set of topological feedback $g_{i j} s$.

Proof:. The proof follows by collecting the results leading to inequalities (13).

In practical designs, computational efficiency can be further improved; e.g., noting that $C_{i}=s_{i 0} \widehat{C}_{i}, s_{i 0}$ is the corresponding topology; hence they are unities for the nodes where vibration sources enter the structure, and they are nil for the nodes without disturbances. For the nodes subject to disturbances, the vibration sources are often independent; then $C_{\mathrm{va}}$ and $C_{\mathrm{ve}}$ can be expressed as $C_{\mathrm{va}}=I_{M, 1}$ and $C_{\mathrm{ve}}=$ $I_{N-M, 0}$ or $C_{\mathrm{va}}=I_{M, 0}$ and $C_{\mathrm{ve}}=I_{N-M, 1}$, depending on the nodes to be attenuated or harvested. The notation $I_{M, 1}=$ $\left[\begin{array}{lll}1 & \cdots & 1\end{array}\right]^{T}$ is for $M$ unity elements and $I_{M, 0}=\left[\begin{array}{ccc}0 & \cdots & 0\end{array}\right]^{T}$ $M$ nil elements, respectively. Consider the situation where the nodes with vibration source are attenuated; then (13) becomes

$$
\begin{array}{r}
\left\|A_{11}^{-1}\left[\left(I+A_{12} P_{22} A_{21} A_{11}^{-1}\right)\right] I_{M, 1}\right\|_{\text {ew }}<\delta_{1}, \\
\left\|P_{22} A_{21} A_{11}^{-1} I_{M, 1}\right\|_{\text {ew }}>\delta_{2} .
\end{array}
$$

Further, consider the specification is prescribed as

$$
\delta_{1}=\frac{1}{\delta_{2}}=\delta
$$

Then, (14) is rewritten as

$$
\begin{array}{r}
\left\|A_{11}^{-1}\left[\left(I+A_{12} P_{22} A_{21} A_{11}^{-1}\right)\right] I_{M, 1}\right\|_{\text {ew }}<\delta, \\
\left\|P_{22} A_{21} A_{11}^{-1} I_{M, 1}\right\|_{\text {ew }}>\frac{1}{\delta} .
\end{array}
$$


Note that, in the above inequalities, multiplication by $I_{M, 1}$ is simply the sum of the row elements for each row of the matrix; computational efficiency can be immensely improved.

This is summarized as follows.

Theorem 2. For a given specification $[\delta(1 / \delta)]^{T}$, active vibration distribution through restricted topology control can be resolved by solving inequalities (16) for a set of topological feedback $g_{i j} s$.

Proof:. The proof follows by collecting the results leading to inequalities (16).

Remark 1. Condition (16) can also be utilized for answering important problem of feasibility of particular specification $[\delta(1 / \delta)]^{T}$; for example, it is possible to attenuate some nodes by $20 \mathrm{~dB}$ while harvesting others by $20 \mathrm{~dB}$ simultaneously.

Remark 2. For the simple case of 2-node structure, (16) can be readily written down as follows:

$$
\begin{aligned}
& \left|\frac{a_{2}-j \omega}{\left(a_{1}-j \omega\right)\left(a_{2}-j \omega\right)-\bar{s}_{12} \bar{s}_{21}}\right|<\delta, \\
& \left|\frac{\bar{s}_{12}}{\left(a_{1}-j \omega\right)\left(a_{2}-j \omega\right)-\bar{s}_{12} \bar{s}_{21}}\right|>\frac{1}{\delta},
\end{aligned}
$$

where $\bar{s}_{12}=s_{12}+g_{12}$ and $\bar{s}_{21}=s_{21}+g_{21}$. Hence, the feedback control $g_{12}$ and $g_{21}$ can be determined for the given system parameters and specification $[\delta(1 / \delta)]^{T}$, provided (17) is solvable. Should (17) be nonsolvable, it implies that the corresponding specification is not achievable.

\section{Active Single Node Control}

In the above formulation, active restricted control is fulfilled through full topology feedback; e.g., all elements $g_{i j} s$ in $G$ are available for feedback. This implies that sensors are required for all the nodes for implementation of the control system. This is not desirable and one question arises whether the number of sensors can be reduced; e.g., is it possible to achieve required performance by using only one node topology feedback?

To develop this important story, a scenario is assumed where the first node is to be attenuated while the remaining nodes are for harvesting. Then it is found $C_{\mathrm{va}}=0$ and $C_{\mathrm{ve}}=I_{N-1,1}$, with $A_{11}=a_{1}-j \omega$. Hence, condition (13) now reads the following:

$$
\begin{gathered}
\frac{\left|A_{12} P_{22} I_{N-1,1}\right|}{\sqrt{a_{1}^{2}+\omega^{2}}}<\delta_{1}, \\
\left\|P_{22} I_{N-1,1}\right\|_{\text {ew }}>\delta_{2},
\end{gathered}
$$

where $P_{22}=\left(A_{22}-\left(A_{21} A_{12} / a_{1}-j \omega\right)\right)^{-1}$

$A_{22}=\left[\begin{array}{ccc}\left(a_{2}-j \omega\right) & \cdots & s_{2 N} \\ \vdots & \ddots & \vdots \\ s_{N 2} & \cdots & \left(a_{N}-j \omega\right)\end{array}\right] ; \quad A_{12}=\left[\begin{array}{c}s_{12}+g_{12} \\ \vdots \\ s_{1 N}+g_{1 N}\end{array}\right]^{T}$; and $A_{21}=\left[\begin{array}{c}s_{21} \\ \vdots \\ s_{N 1}\end{array}\right]$.

A performance specification $[\delta(1 / \delta)]^{T}$ thus leads to

$$
\begin{gathered}
\frac{\left|A_{12} P_{22} I_{N-1,1}\right|}{\sqrt{a_{1}^{2}+\omega^{2}}}<\delta, \\
\left\|P_{22} I_{N-1,1}\right\|_{\text {ew }}>\frac{1}{\delta} .
\end{gathered}
$$

This is summarized in the following.

Lemma 3. For specification $[\delta(1 / \delta)]^{T}$, active vibration distribution through restricted single node control can be resolved by solving inequalities (19) for feedback controls $g_{1 j}$, $j \in[2, N]$.

Proof:. The proof follows by collecting the results leading to inequalities (19).

Remark 3. It is noted that, for a frequency $\omega$, the first inequality in (19) can be manipulated to be

$$
\left|A_{12}\left(P_{22} I_{N-1,1}\right)\right|<\delta \sqrt{a_{1}^{2}+\omega^{2}} .
$$

The term within the bracket is exactly the same as the left part in the second inequality.

Define

$$
V=P_{22} I_{N-1,1}=\left[\begin{array}{lll}
v_{1} & \cdots & v_{N-1}
\end{array}\right]^{T} .
$$

Then, (19) is simply equivalent to the following $N$-simultaneous inequalities:

$$
\begin{aligned}
\left|\sum_{i=1}^{N-1} v_{i}\left(s_{1(i+1)}+g_{1(i+1)}\right)\right| & <\delta \sqrt{a_{1}^{2}+\omega^{2}}, \\
\left|v_{i}\right| & >\frac{1}{\delta}, \\
i & \in[1, \ldots, N-1] .
\end{aligned}
$$

This observation can be used for simplifying the corresponding calculation.

Remark 4. It is expected that severe restriction to the parameters $g_{1 j}, j \in[2, N]$ can exist which might render the specification $[\delta(1 / \delta)]^{T}$ nonfeasible. Consequently, performance specification must be relaxed for active restricted single node control to be possible.

Remark 5. The above solution leads to a nonsymmetric topology for closed-loop control system. Hence, should a symmetric or nondirectional topology be enforced, the only modification is simply to replace $A_{21}$ with 
$A_{21}^{T}=\left[s_{12}+g_{12} \cdots s_{1 N}+g_{1 N}\right]=A_{12}$. These constrained cases are detailed in the next section.

\section{Active Single Node Control: Constraints and Robustness Consideration}

Any realistic system will contain constraints in one form or another. They can be roughly classified into system constraints and parameter constraints. The latter refers to design parameters such as $g_{i j} s$ being constrained, while the former refers to system states, system outputs, etc. being bounded. As the system states and outputs can be expressed as a function of parameters, it is of convenience to express system constraints as bounded parameters:

$$
\begin{aligned}
\vec{g}_{i j} \leq g_{i j} \leq \overleftarrow{g}_{i j}, \quad \forall i \in[1, \ldots, N], \\
j \in[1, \ldots, N] \text { and } i \neq j,
\end{aligned}
$$

where $\vec{g}_{i j}$ and $\overleftarrow{ }_{i j}$ denote the lower and upper limits of $g_{i j}$. Similarly, parameter uncertainty can also be expressed as additive uncertainty $\Delta_{i j}$ :

$$
\begin{aligned}
g_{i j}-\Delta_{i j} \leq g_{i j} \leq g_{i j}+\Delta_{i j}, \quad \forall i \in[1, \ldots, N], & \\
& j \in[1, \ldots, N] i \neq j .
\end{aligned}
$$

Consequently, the issue of constraint handling and robustness can be tackled by "incorporating" (23) and (24) into the active restricted control formulation, for example, for restricted single node control.

Lemma 4. For specification $[\delta(1 / \delta)]^{T}$, active vibration distribution through restricted single node control with constraints can be resolved by jointly solving inequalities (19), (23), and (24) for feedback controls $g_{1 j}, j \in[2, \ldots, N]$.

Proof:: the proof follows by collecting the results leading to inequalities (24).

The above result can certainly be applied to situations where some $g_{i j} s$ is zero; for example, there is no topological connection among certain nodes. This can be developed into its extreme case by assuming that only one element is available for feedback. From (5) and (10)-(12), the following can be obtained:

$$
P_{22}=\left[\begin{array}{cccc}
\left(a_{2}-j \omega\right)-s_{21}\left(s_{12}+g_{12}\right) & s_{23}-s_{21}\left(s_{13}+g_{13}\right) & \cdots & s_{2 N}-s_{21}\left(s_{1 N}+g_{1 N}\right) \\
s_{32}-s_{31}\left(s_{12}+g_{12}\right) & \left(a_{3}-j \omega\right)-s_{31}\left(s_{13}+g_{13}\right) & \cdots & s_{3 N}-s_{31}\left(s_{1 N}+g_{1 N}\right) \\
\vdots & \vdots & \ddots & \vdots \\
s_{N 2}-s_{N 1}\left(s_{12}+g_{12}\right) & s_{N 3}-s_{N 1}\left(s_{13}+g_{13}\right) & \cdots & \left(a_{N}-j \omega\right)-s_{N 1}\left(s_{1 N}+g_{1 N}\right)
\end{array}\right]^{-1}
$$

Without loss of generality, it is assumed that $g_{12}$ is to be designed; then, (25) leads to

$$
P_{22}=\left[\begin{array}{cccc}
\left(a_{2}-j \omega\right)-s_{21}\left(s_{12}+g_{12}\right) & s_{23}-s_{21} s_{13} & \cdots & s_{2 N}-s_{21} s_{1 N} \\
s_{32}-s_{31}\left(s_{12}+g_{12}\right) & \left(a_{3}-j \omega\right)-s_{31} s_{13} & \cdots & s_{3 N}-s_{31} s_{1 N} \\
\vdots & \vdots & \ddots & \vdots \\
s_{N 2}-s_{N 1}\left(s_{12}+g_{12}\right) & s_{N 3}-s_{N 1} s_{13} & \cdots & \left(a_{N}-j \omega\right)-s_{N 1} s_{1 N}
\end{array}\right]^{-1}
$$

Decomposition of $P_{22}$ leads to

$$
P_{22}=\left\{\left[\begin{array}{ccc}
\left(a_{2}-j \omega\right)-s_{21} s_{12} & \cdots & s_{2 N}-s_{21} s_{1 N} \\
\vdots & \ddots & \vdots \\
\left.s_{N 2}-s_{N 1} s_{12}\right) & \cdots & \left(a_{N}-j \omega\right)-s_{N 1} s_{1 N}
\end{array}\right]+\left[\begin{array}{cccc}
-s_{21} g_{12} & 0 & \cdots & 0 \\
\vdots & 0 & \ddots & \vdots \\
-s_{N 1} g_{12} & 0 & \cdots & 0
\end{array}\right]\right\}^{-1} P_{22}=(M+N)^{-1} .
$$

Define

$$
\begin{aligned}
& M=\left[\begin{array}{ccc}
\left(a_{2}-j \omega\right)-s_{21} s_{12} & \cdots & s_{2 N}-s_{21} s_{1 N} \\
\vdots & \ddots & \vdots \\
\left.s_{N 2}-s_{N 1} s_{12}\right) & \cdots & \left(a_{N}-j \omega\right)-s_{N 1} s_{1 N}
\end{array}\right] \\
& N=\left[\begin{array}{cccc}
-s_{21} g_{12} & 0 & \cdots & 0 \\
\vdots & 0 & \ddots & \vdots \\
-s_{N 1} g_{12} & 0 & \cdots & 0
\end{array}\right] \text {; then, }
\end{aligned}
$$

and

Utilization of the Woodbury matrix identity leads to

$$
P_{22}=M^{-1}-M^{-1}\left(I+\mathrm{NM}^{-1}\right)^{-1} M^{-1} .
$$

Thus, the terms in (19) can be manipulated to be 


$$
\begin{aligned}
P_{22} I_{N-1,1} & =M^{-1} I_{N-1,1}-M^{-1}\left(I+N M^{-1}\right)^{-1} M^{-1} I_{N-1,1}, \\
A_{12} P_{22} I_{N-1,1} & =\left[\begin{array}{lll}
s_{12}+g_{12} & \cdots & s_{1 N}
\end{array}\right] M^{-1} I_{N-1,1}-\left[\begin{array}{lll}
s_{12}+g_{12} & \cdots & s_{1 N}
\end{array}\right] M^{-1}\left(I+N M^{-1}\right)^{-1} M^{-1} I_{N-1,1} .
\end{aligned}
$$

Therefore inequalities in (19) are equivalent to

$$
\begin{aligned}
& \frac{\left|\left[s_{12}+g_{12} \cdots s_{1 N}\right] M^{-1} I_{N-1,1}-\left[s_{12}+g_{12} \cdots s_{1 N}\right] M^{-1}\left(I+N M^{-1}\right)^{-1} M^{-1} I_{N-1,1}\right|}{\sqrt{a_{1}^{2}+\omega^{2}}}<\delta, \\
& \left\|M^{-1} I_{N-1,1}-M^{-1}\left(I+N M^{-1}\right)^{-1} M^{-1} I_{N-1,1}\right\|_{\text {ew }}>\frac{1}{\delta} \text {. }
\end{aligned}
$$

Now, it is seen that all the elements in matrix $M$ are known; henceforth, its inverse can be precomputed; the design parameter $g_{12}$ is associated with the matrix $N$, which is sparse and has elements only in the first column with all others being nil, facilitating with the computations.

The above development is summarized in the following.

Lemma 5. For specification $[\delta(1 / \delta)]^{T}$, active vibration distribution through restricted single node and single element control can be resolved by solving inequalities (31) for feedback gain $g_{12}$.

Remark 6. Albeit usual, the above extreme situation is necessitated where the environment is harsh and system implementation should be kept simple. Indeed, should the simplest solution be feasible, it is also desirable to avoid complicated sensing and actuation devices.

Remark 7. Given the above exposition, it would thus be interesting and useful to consider the problem of optimal selection of single element feedback control. Such an active restricted single node and single element control makes a contribution to the current solutions.

Remark 8. It is also worth pointing out that the proposal methodology can be readily combined with those model-free methods such as fuzzy logic controls for dealing with large operational envelope control problems.

\section{Restricted Active Topology Control: Performance Limit}

The above developments are essentially "solvability" conditions for restricted active topology control. The above theoretical results thus provide solutions for the corresponding assumed scenarios for an "arbitrarily" chosen performance specification. If a null set results from the related conditions, it will imply that no designs or appropriate $g_{i j}$ can meet the designated performance specification $\left[\begin{array}{ll}\delta_{1} & \delta_{2}\end{array}\right]^{t}$. Consequently the specification must be relaxed to permit the existence of solutions. The appearance of solutions in $g_{i j}$ certainly indicates the best achievable performance for relaxed specification.
On the contrary, existence of $g_{i j}$ means that the designated performance specification $\left[\begin{array}{ll}\delta_{1} & \delta_{2}\end{array}\right]^{T}$ can be satisfied. Thus, performance can be further improved by decreasing $\delta_{1}$ for more attenuation and increasing $\delta_{2}$ for more harvesting. The disappearance of solutions in $g_{i j}$ certainly indicates the best achievable performance for enhanced specification.

For both scenarios, detailed adjustment in $\delta_{1}$ and $\delta_{2}$ can be complex since they are defined by vectors $\delta_{1}=\left[\begin{array}{llll}\delta_{1,1} & \cdots & \delta_{1, M}\end{array}\right]^{T}$ and $\delta_{2}=\left[\begin{array}{llll}\delta_{2,(M+1)} & \cdots & \delta_{2, N}\end{array}\right]^{T}$. Clear-cut conditions can be obtained for simple dynamical systems; however, when vibration needs to be distributed over a large number of nodes, trials and errors are involved for approaching the performance limit. But the development here does provide guidance for performance improvement towards fundamental limit.

Indeed, fundamental limit does exist which is independent of parameters to be designed. Still consider the 2node example in Remark 2; suppose the system parameters $a_{1}, a_{2}, s_{12}$, and $s_{21}$ are given while the to-be-designed parameters are constrained (due to physical implementation, size, weight, or whatever reasons) such that

$$
\begin{array}{r}
\left|a_{1}-j \omega\right| \mid<\frac{1}{2 \delta}, \\
\frac{\left|s_{12}+g_{12}\right|\left|s_{21}+g_{21}\right|}{\left|a_{2}-j \omega\right|}<\frac{1}{2 \delta} .
\end{array}
$$

The above inequalities can be fulfilled considering the fact that $\delta$ is often expected to be a very small constant far less than unity. Then, the following holds:

$$
\frac{\left|s_{12}+g_{12}\right|\left|s_{21}+g_{21}\right|}{\left|a_{2}-j \omega\right|}+\left|a_{1}-j \omega\right|<\frac{1}{\delta} .
$$

Consequently, we must have

$$
\frac{\left|\left(a_{1}-j \omega\right)\left(a_{2}-j \omega\right)-\left(s_{12}+g_{12}\right)\left(s_{21}+g_{21}\right)\right|}{\left|a_{2}-j \omega\right|}<\frac{1}{\delta} .
$$

That is,

$$
\left|\frac{a_{2}-j \omega}{\left(a_{1}-j \omega\right)\left(a_{2}-j \omega\right)-\left(s_{12}+g_{12}\right)\left(s_{21}+g_{21}\right)}\right|>\delta,
$$


or

$$
\left|\frac{a_{2}-j \omega}{\left(a_{1}-j \omega\right)\left(a_{2}-j \omega\right)-\bar{s}_{12} \bar{s}_{21}}\right|>\delta .
$$

This is in a direct contradiction with the required specification:

$$
\left|\frac{a_{2}-j \omega}{\left(a_{1}-j \omega\right)\left(a_{2}-j \omega\right)-\bar{s}_{12} \bar{s}_{21}}\right|<\delta .
$$

For $\delta=0.5$, this simply says that it is not possible to attenuate node 1 by $6 \mathrm{~dB}$ through any feedback design; henceforth, a fundamental performance limit is enforced.

Similarly, if

$$
\left|\frac{\left(a_{1}-j \omega\right)\left(a_{2}-j \omega\right)}{s_{12}+g_{12}}-s_{21}-g_{21}\right|>\delta
$$

holds, then it will lead to

$$
\left|\frac{s_{12}+g_{12}}{\left(a_{1}-j \omega\right)\left(a_{2}-j \omega\right)-\left(s_{12}+g_{12}\right)\left(s_{21}+g_{21}\right)}\right|<\frac{1}{\delta} .
$$

This is conflicting with the performance specification:

$$
\left|\frac{\bar{s}_{12}}{\left(a_{1}-j \omega\right)\left(a_{2}-j \omega\right)-\bar{s}_{12} \bar{s}_{21}}\right|>\frac{1}{\delta},
$$

with $\delta=0.25$; this implies that enhancement of node 2 by $12 \mathrm{~dB}$ is not possible through any feedback design. Combining the above arguments, it is clearly seen that performance limit exists for vibration distribution, which puts fundamental constraints on vibration attenuation as well as vibration harvesting. These constraints must be respected by any performance specification through any design.

Remark 9. Indeed, fundamental limit problem should be considered for any practical design. This is particularly true for active vibration distribution since not only the indices within $\delta_{1,1} \quad \cdots \quad \delta_{1, M}$ and $\delta_{2,(M+1)} \quad \cdots \quad \delta_{2, N}$, but also $\delta_{1}$ and $\delta_{2}$ themselves can be "conflicting" implying that performance is improved for one set of nodes with the other set's performance having to be deteriorated. Different from conventional conflicting requirements, the fundamental limit discussed here is not conquered by any design. They must be regarded as benchmarking for performance specification and measure of "distance" from best achievable performance.

\section{Numerical Examples}

Consider a 3-node system with the first two nodes subject to an exogenous vibration:

$$
\dot{x}_{i}=a_{i} x_{i}+\sum_{\substack{j=1 \\ j \neq i}}^{3} s_{i j} x_{j}-d_{k}(t), \quad i \in[1,2,3], k=1,2,
$$

where the underlying graph with nodes dynamics and topological interaction can be compactly written as
$P=\left[\begin{array}{lll}a_{1} & s_{12} & s_{13} \\ s_{21} & a_{2} & s_{23} \\ s_{31} & s_{32} & a_{3}\end{array}\right]$. To be specific, $P$ is taken as $p=\left[\begin{array}{ccc}-1 & 1 & 0 \\ 1 & -3 & 1 \\ 0 & 1 & -2\end{array}\right]$. A scenario can now be assumed where the first two nodes are required to be attenuated by at least $6 \mathrm{~dB}$ and $3 \mathrm{~dB}$, respectively, and the last node for harvesting by at least $6 \mathrm{~dB}$, yet only through restricted active topology feedback. That is, the objective is to achieve the specification $\left[\begin{array}{ll}\delta_{1} & \delta_{2}\end{array}\right]^{T}$ though the design of $u_{i}(t)=\sum_{j=1}^{3} g_{i j} x_{j}(t)$ with

$$
\begin{aligned}
\delta_{1} & =\left[\begin{array}{c}
0.5 \\
0.707
\end{array}\right], \\
\delta_{2} & =2, \\
G & =\left[\begin{array}{ccc}
0 & g_{12} & g_{13} \\
g_{21} & 0 & g_{23} \\
g_{31} & g_{32} & 0
\end{array}\right] .
\end{aligned}
$$

7.1. Active Vibration Distribution through Restricted Topology Control. Theorem 1 dictates that the design objective can be achieved provided that condition (13) is fulfilled. Theorem 2 specifies Theorem 1 with $C_{\mathrm{va}}=I_{2,1}, C_{\mathrm{ve}}=I_{1,0}$ which is exactly the situation here. Henceforth, from Theorem 2, condition (16) must be checked for the design $g_{i j} s$. That is,

$$
\begin{gathered}
\left\|A_{11}^{-1}\left[\left(I+A_{12} P_{22} A_{21} A_{11}^{-1}\right)\right] I_{2,1}\right\|_{\text {ew }}<\left[\begin{array}{c}
0.5 \\
0.707
\end{array}\right], \\
\left|P_{22} A_{21} A_{11}^{-1} I_{2,1}\right|>2 .
\end{gathered}
$$

Now one has

$$
\begin{aligned}
& A_{11}=\left[\begin{array}{cc}
-1-j \omega & 1+g_{12} \\
1+g_{21} & -3-j \omega
\end{array}\right], \\
& A_{12}=\left[\begin{array}{c}
g_{13} \\
1+g_{23}
\end{array}\right], \\
& A_{21}=\left[\begin{array}{c}
g_{31} \\
1+g_{32}
\end{array}\right]^{T}, \\
& A_{22}=-2-j \omega .
\end{aligned}
$$

The inequalities in (43) can be readily computed off leading to

$$
\begin{aligned}
\left|\frac{p_{1}+p_{2}}{\Delta_{1} \Delta_{2}}\right| & <0.5, \\
\left|\frac{p_{3}+p_{4}}{\Delta_{1} \Delta_{2}}\right| & <0.707, \\
\left|\frac{p_{5}}{\Delta_{2}}\right| & >2,
\end{aligned}
$$

where 


$$
\begin{aligned}
\Delta_{1}= & 3-\omega^{2}-\left(1+g_{12}\right)\left(1+g_{21}\right)+4 j \omega, \\
\Delta_{2}= & 2\left(1+g_{12}\right)^{2}+\left(1+g_{12}\right)\left(1+g_{32}\right) g_{13}+\left(1+g_{21}\right)\left(1+g_{23}\right) g_{31}+\left(1+g_{32}\right)\left(1+g_{23}\right) \\
& +3 g_{13} g_{31}+6 \omega^{2}-6+j\left[\left(1+g_{23}\right)\left(1+g_{32}\right)+g_{13} g_{31}+\left(1+g_{12}\right)^{2} \omega+\omega^{3}-11 \omega\right], \\
p_{1}= & (3+j \omega)\left[-\Delta_{2}+\left(1+g_{12}\right)\left(1+g_{32}\right) g_{13}+3 g_{13} g_{31}+j g_{13} g_{31} \omega\right] \\
& +\left(1+g_{12}\right)\left[\left(1+g_{21}\right)\left(1+g_{23}\right)\left(1+g_{32}\right)+3\left(1+g_{32}\right) g_{31}+j\left(1+g_{32}\right) g_{31} \omega\right], \\
p_{2}= & (3+j \omega)\left[\left(1+g_{12}\right) g_{31} g_{13}+\left(1+g_{32}\right) g_{13}+j\left(1+g_{32}\right) g_{13} \omega\right] \\
& +\left(1+g_{12}\right)\left[-\Delta_{2}+\left(1+g_{12}\right)\left(1+g_{23}\right) g_{31}+\left(1+g_{23}\right)\left(1+g_{32}\right)+j\left(1+g_{23}\right)\left(1+g_{32}\right) \omega\right], \\
p_{3}= & (3+j \omega)\left[\left(1+g_{12}\right)\left(1+g_{23}\right)\left(1+g_{32}\right)+3\left(1+g_{32}\right) g_{31}+j\left(1+g_{32}\right) g_{31} \omega\right] \\
& +\left(1+g_{21}\right)\left[-\Delta_{2}+\left(1+g_{12}\right)\left(1+g_{32}\right) g_{13}+3 g_{31} g_{13}+j g_{31} g_{13} \omega\right], \\
p_{4}= & (3+j \omega)\left[-\Delta_{2}+\left(1+g_{12}\right)\left(1+g_{23}\right) g_{31}+\left(1+g_{23}\right)\left(1+g_{32}\right)+j\left(1+g_{23}\right)\left(1+g_{32}\right) \omega\right] \\
& +\left(1+g_{12}\right)\left[\left(1+g_{12}\right) g_{31} g_{13}+\left(1+g_{32}\right) g_{13}+j\left(1+g_{32}\right) g_{13} \omega\right], \\
p_{4}= & (3+j \omega)\left[-\Delta_{2}+\left(1+g_{12}\right)\left(1+g_{23}\right) g_{31}+\left(1+g_{23}\right)\left(1+g_{32}\right)+j\left(1+g_{23}\right)\left(1+g_{32}\right) \omega\right] \\
& +\left(1+g_{12}\right)\left[\left(1+g_{12}\right) g_{31} g_{13}+\left(1+g_{32}\right) g_{13}+j\left(1+g_{32}\right) g_{13} \omega\right] .
\end{aligned}
$$

A straightforward calculation for the inequalities in (45) with a symmetric graph $G$ at unity frequency results in conditions where the to-be-designed parameters $g_{12}, g_{13}$, and $g_{23}$ satisfy

$$
\begin{array}{r}
\frac{\left|q_{1}\right|}{|\Delta|\left|2-\left(1+g_{12}\right)^{2}+4 j\right|}<0.5, \\
\frac{\left|q_{2}\right|}{|\Delta|\left|2-\left(1+g_{12}\right)^{2}+4 j\right|}<0.707, \\
\frac{\left|\left(2+g_{12}\right)\left(1+g_{23}\right)+\left(1+g_{23}\right) g_{13}+3 g_{13}+j\left(1+g_{13}+g_{23}\right)\right|}{|\Delta|}>2,
\end{array}
$$

with

$$
\begin{aligned}
\Delta= & 2\left(1+g_{12}\right)^{2}+2\left(1+g_{12}\right)\left(1+g_{23}\right) g_{13}+\left(1+g_{23}\right)^{2}+3 g_{13}^{2}+j\left[\left(1+g_{23}\right)^{2}+g_{13}^{2}+\left(1+g_{12}\right)^{2}-10\right] \\
q_{1}= & -2\left(1+g_{12}\right)^{3}-5\left(1+g_{12}\right)^{2}-2\left(1+g_{23}\right)^{2}+2\left(1+g_{23}\right) g_{13}-\left(1+g_{12}\right)^{2}\left(1+g_{23}\right) g_{13} \\
& +\left(1+g_{12}\right)^{2}\left(1+g_{23}\right)^{2}-10+j\left[-\left(1+g_{12}\right)^{3}-5\left(1+g_{12}\right)^{2}-4\left(1+g_{23}\right)^{2}+4\left(1+g_{23}\right) g_{13}+10\left(1+g_{12}\right)\right] ; \\
q_{2}= & -2\left(1+g_{12}\right)^{3}-5\left(1+g_{12}\right)^{2}-\left(1+g_{12}\right)^{2}\left(1+g_{23}\right) g_{13}+\left(1+g_{12}\right)^{2} g_{13}^{2}-2\left(1+g_{12}\right)\left(1+g_{23}\right) g_{13}+2\left(1+g_{12}\right)\left(1+g_{23}\right)^{2} \\
& +8\left(1+g_{23}\right) g_{13}-8 g_{13}^{2}-10+j\left[-\left(1+g_{12}\right)^{3}-5\left(1+g_{12}\right)^{2}+6\left(1+g_{23}\right) g_{13}-6 g_{13}^{2}+10\left(1+g_{12}\right)+30\right] .
\end{aligned}
$$

7.2. Active Single Node Control for Vibration Distribution. Inequalities in (47) are the general conditions that $g_{12}$, $g_{13}$, and $g_{23}$ must satisfy to fulfill the performance specification $\left[\begin{array}{lll}0.5 & 0.707 & 2\end{array}\right]^{T}$. Direct calculation seems to be complicated but it is not as daunting as they look, and, quite usual, significant simplification can be achieved by assuming special values for the graph topology, e.g., $g_{23}=-1$. Indeed, this implies $g_{23}+1=0$, simply indicating that the connection between node 2 and node 3 is broken up. Therefore, it essentially leads to a restricted single node control solution. Now, (47) can be written as 


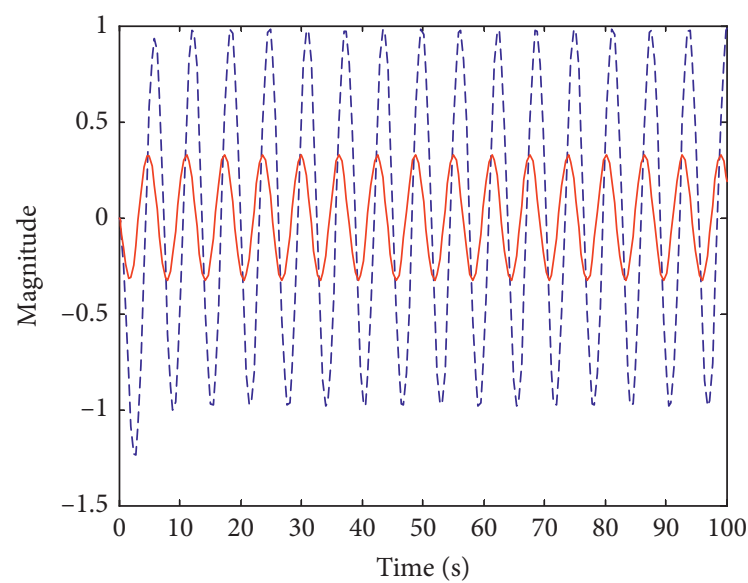

- - - Open-loop performance

- Closed-loop performance

(a)

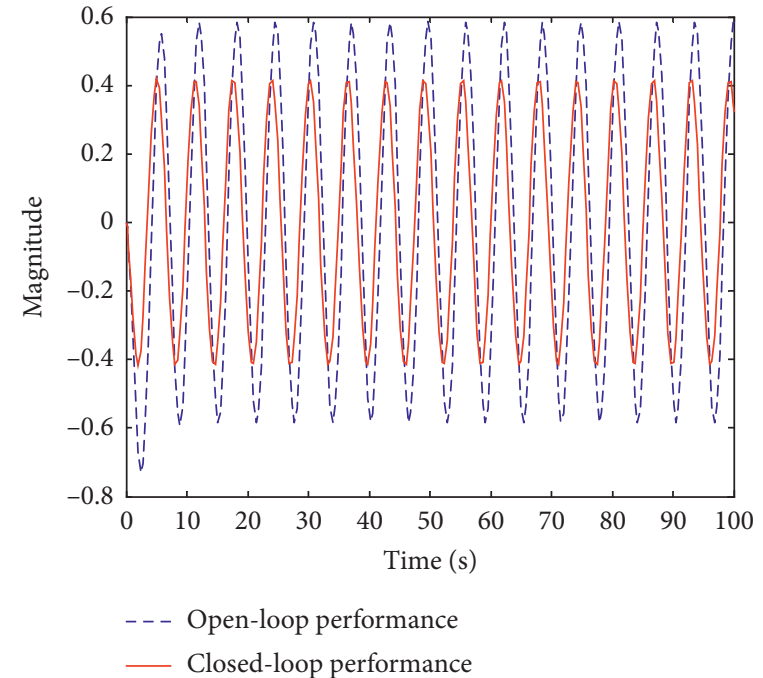

(b)

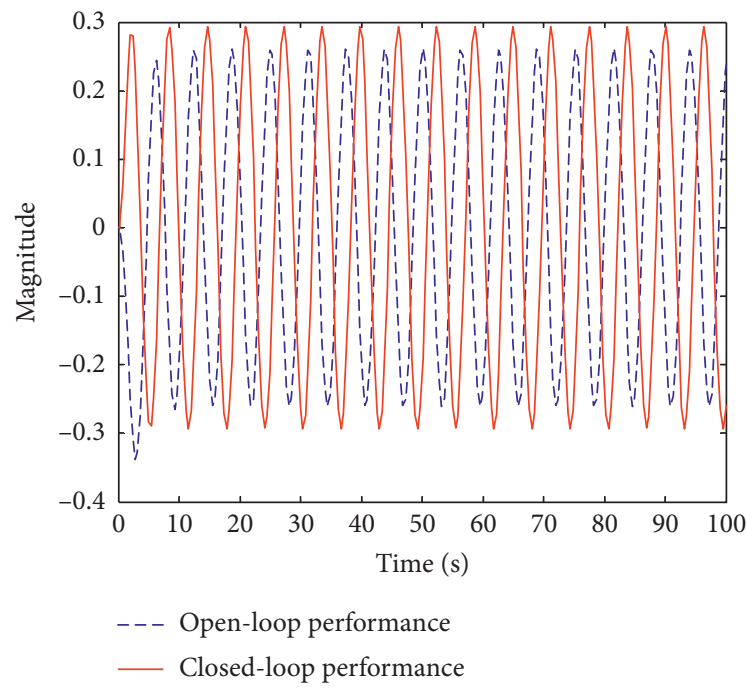

(c)

FiguRE 1: Restricted active vibration distribution through single node control: (a) node 1 performance; (b) node 2 performance; (c) node 3 performance.

$$
\begin{gathered}
\frac{\left|-2\left(1+g_{12}\right)^{3}-5\left(1+g_{12}\right)^{2}-10+j\left[-\left(1+g_{12}\right)^{3}-5\left(1+g_{12}\right)^{2}+10\left(1+g_{12}\right)\right]\right|}{|\Delta|\left|2-\left(1+g_{12}\right)^{2}+4 j\right|}<0.5, \\
\frac{\left|\left(g_{13}^{2}-2 g_{12}-7\right)\left(1+g_{12}\right)^{2}-8 g_{13}^{2}-10+j\left[-\left(6+g_{12}\right)\left(1+g_{12}\right)^{2}-6 g_{13}^{2}+10 g_{12}+40\right]\right|}{|\Delta|\left|2-\left(1+g_{12}\right)^{2}+4 j\right|}<0.707 \\
\frac{\left|10 g_{13}\right|}{\sqrt{5\left(1+g_{12}\right)^{4}+\left(1+g_{12}\right)^{2}\left(14 g_{13}^{2}-20\right)+9 g_{13}^{2}+\left(g_{13}^{2}-10\right)^{2}}}>2 .
\end{gathered}
$$




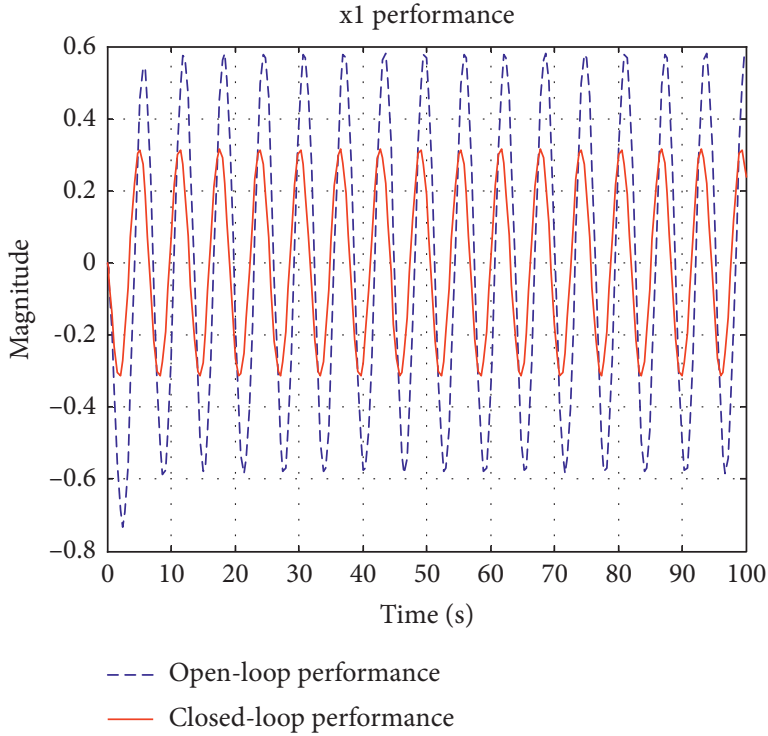

(a)

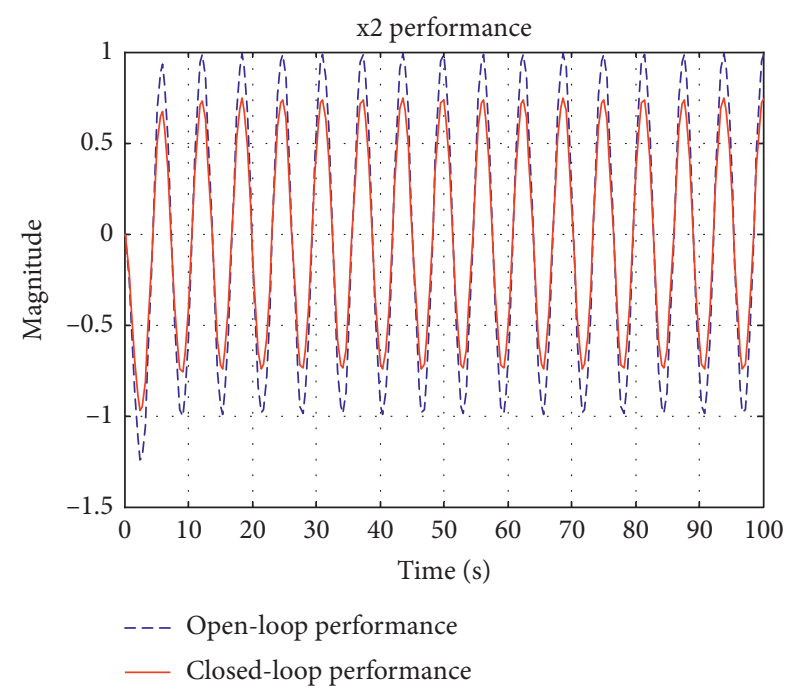

(b)

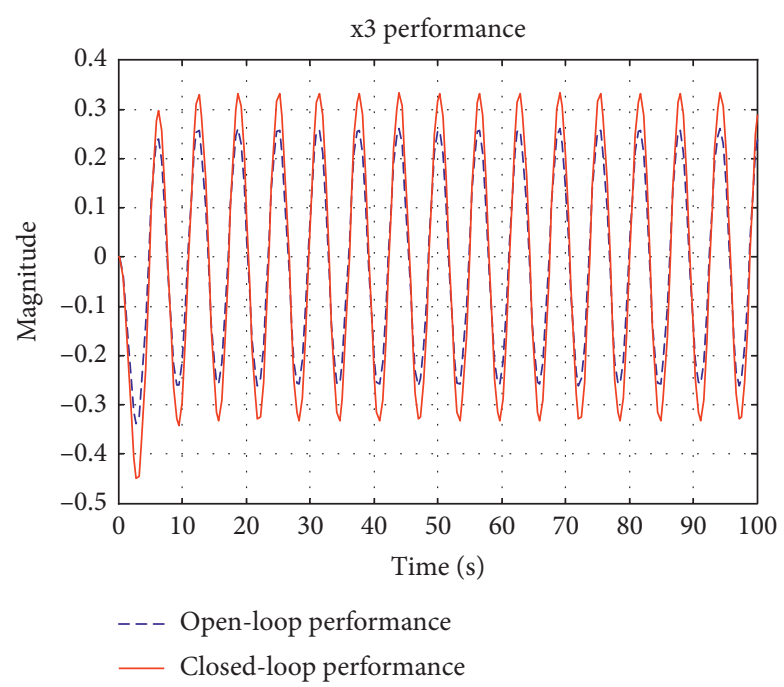

(c)

FIGURE 2: Restricted active vibration distribution through single node and single element control: (a) node 1 performance; (b) node 2 performance; (c) node 3 performance.

This single node control solution can be readily checked out by further assuming $g_{12}=-2$, resulting in

$$
\begin{gathered}
\frac{|13+14 j|}{\left|2+3 g_{13}^{2}+j\left(g_{13}^{2}-9\right)\right||1+4 j|}<0.5, \\
\frac{\left|13+7 g_{13}^{2}+j\left(6 g_{13}^{2}-16\right)\right|}{\left|2+3 g_{13}^{2}+j\left(g_{13}^{2}-9\right)\right||1+4 j|}<0.707, \\
\frac{\left|10 g_{13}\right|}{\sqrt{23 g_{13}^{2}-15+\left(g_{13}^{2}-10\right)^{2}}}>2 .
\end{gathered}
$$

It can be readily checked that the first inequality is always satisfied; the second inequality requires $g_{13}^{2}<7.26$, while the third inequality leads to the solution $5<g_{13}^{2}<17$. Henceforth, the final solution for $g_{13}$ is the intersection leading to

$$
5<g_{13}^{2}<7.26 \text {. }
$$

To sum up, the performance requirement can be achieved through active single node control with $g_{12}=-2$ and $5<g_{13}^{2}<7.26$. To validate this situation, a real-time simulation should be carried out. However a stability check shows that $g_{13}^{2}<1.3$ and the original performance specification is not feasible, and a performance loss must be accepted. A choice of $g_{13}=1$ is now carried out with the performance shown in Figure 1. It is seen that a $10 \mathrm{~dB}$ and $3.5 \mathrm{~dB}$ reduction in nodes 1 and 2, respectively, and $1.6 \mathrm{~dB}$ enhancement in node 3 are achieved. Although there is a loss of performance in node 3 , the performance in both node 1 and node 2 has been achieved 
better than the specification. This exemplifies Lemma 3 for active single node control in Section 4 .

7.3. Active Restricted Single Node Control: Parametric Constraints and Performance Limit. For the above single node control solution, the required $g_{13}$ can be further solved from (51) as follows:

$$
g_{13} \in\left[\begin{array}{ll}
-2.7 & -2.2
\end{array}\right] \cup\left[\begin{array}{ll}
2.2 & 2.7
\end{array}\right] .
$$

From continuity of solutions, it is known that for $g_{12} \in[-2-\varepsilon-2+\varepsilon]$ with $\varepsilon$ being a very small positive number, $g_{13}$ will wander around the neighborhood of the sets (52). This roughly provides the ranges of $g_{12}$ and $g_{13}$ for performance specification to be satisfied. Therefore, if the parameter values are bounded out of the ranges, the specification has to be modified. This is the constrained single node solution expounded in Lemma 4. For Lemma 5 for active single node and single element control, either $g_{12}=$ -1 or $g_{13}=0$ needs to be checked.

First, consider $g_{12}=-1$; then, from (49), $g_{13}$ must satisfy

$$
\begin{gathered}
\frac{10}{\left|3 g_{13}^{2}+j\left(g_{13}^{2}-10\right)\right| 2+4 j \mid}<0.5, \\
\frac{\left|8 g_{13}^{2}+10+j\left(6 g_{13}^{2}-30\right)\right|}{\left|3 g_{13}^{2}+j\left(g_{13}^{2}-10\right)\right| 2+4 j \mid}<0.707, \\
\frac{\left|10 g_{13}\right|}{\left|3 g_{13}^{2}+j\left(g_{13}^{2}-10\right)\right|}>2 .
\end{gathered}
$$

The first inequality can always be satisfied while the second inequality is interesting; since the left-hand side is exactly 0.707 , henceforth, reduction in node 2 is always $3 \mathrm{~dB}$ for any $g_{13}$. Thus, should the specification for node 2 be marginally relaxed, the performance can always be achieved. The final solution thus comes from the third inequality with

$$
3<g_{13}^{2}<33 .
$$

For $g_{13}=0$, as no solutions exist for the third inequality in (49), henceforth single node control with $g_{13}=0$ is not possible. It is thus concluded that the performance specification can still be satisfied only through single element $g_{13}$ feedback, provided that it satisfies condition (54). Meanwhile, from the above derivations, it is also noticed that specification for node 1 is always satisfied indicating that performance can be further improved, while for the active single node and single element control, the left-hand side of the second inequality in (53) is 0.707 , implying that the performance limit for node 2 is $3 \mathrm{~dB}$; or the maximum attenuation in node 2 is $3 \mathrm{~dB}$. This illustrates the interrelationship among performance specification, system parameters, and performance limit.

Finally, a real-time simulation is presented where active vibration distribution is achieved through the above single node and single element feedback. However, it must be noticed that the stability of the network requires $\left|g_{13}\right|<\sqrt{2}$, and $g_{13}$ feedback demands $\sqrt{3}<g_{13}<\sqrt{33}$ from (54). It is thus expected that a single node and single element feedback with $\left|g_{13}\right|=1$ will provide marginal loss of performance to specification. The results with $g_{13}=1$ are shown in Figure 2. It is seen that a $5.7 \mathrm{~dB}$ and $2.7 \mathrm{~dB}$ reduction in nodes 1 and 2 , respectively, and $2.3 \mathrm{~dB}$ enhancement in node 3 are achieved. Although the performance is specified as $6 \mathrm{~dB}, 3 \mathrm{~dB}$, and $6 \mathrm{~dB}$, it is still remarkable since the vibration distribution is achieved with a single topological connection between node 1 and node 3 , even significantly simplifying the original system topology.

\section{Conclusion}

The problem of active vibration distribution has been considered. The important issues of solvability with restricted topology, restricted single node, and restricted parameter constraints have been resolved. The closely related performance limits problems have also been given considerations. As a consequence, a systematic design methodology for active restricted vibration distribution through restricted control has been established. The design procedures have been validated through numerical examples.

Finally, it is worth pointing out that vibration distribution problem is generic and pertinent with many engineering designs. For example, vibration can be distributed being attenuated at the engine mount for prevention of propagation to the hull, while being enhanced at other specific locations for high-frequency radiation for stealth purpose; new types of mass dampers can be developed while transforming the vibrational damping force into energy to be harnessed through electrical circuits. The results presented here provide an important proposal with restricted active control solutions.

\section{Data Availability}

The underlying data supporting the results of this study are readily from the author upon request.

\section{Conflicts of Interest}

The author declares no conflicts of interest.

\section{Acknowledgments}

This work was supported by the Fundamental Research Funds for the Central Universities (no. NS2020017), Aviation Science Fund of China-Xi' an 631 Research Institute (no. 201919052001), Central Military Commission Foundation to Strengthen Program Technology Fund (no. 2019-JCJQ-JJ347), Major Special Basic Research Projects for Aviation Engines \& Gas Turbines (no. 1002-DLJ19002), and Major Special Basic Research Projects for Green \& Sustainable Manufacturing (no. 1002-DCB16001).

\section{References}

[1] R. Burdzik, "Monitoring system of vibration propagation in vehicles and method of analysing vibration modes," 
Telematics in the Transport Environment, vol. 329, pp. 406413, 2012.

[2] A. Jha, G. Sahay, and A. Sivaramasastry, "Framework and platform for next generation aircraft health management system," SAE Technical Paper 2017-01-2126, SAE International, Warrendale, PA, USA, 2017.

[3] A. J. Volponi, "Gas turbine engine health management: past, present, and future trends," ASME Journal of Engineering, Gas Turbines \& Power, vol. 136, no. 5, Article ID 051201, 2014.

[4] X. Wu, L. Li, N. Zhou et al., "Diagnosis of DC bias in power transformers using vibration feature extraction and a pattern recognition method," Energies, vol. 11, no. 7, p. 1775, 2018.

[5] B. Garcia, J. C. Burgos, and A. Alonso, "Transformer tank vibration modeling as a method of detecting winding deformations-part I: theoretical foundation," IEEE Transactions on Power Delivery, vol. 21, no. 1, pp. 157-163, 2006.

[6] N. Yang, L.-Y. Chen, H. Yi, and Y. Liu, "A unified solution for vibration analysis of plates with general structural stress distributions," International Journal of Naval Architecture and Ocean Engineering, vol. 8, no. 6, pp. 615-630, 2016.

[7] X. M. Zhang, "Frequency analysis of submerged cylindrical shells with the wave propagation approach," International Journal of Mechanical Sciences, vol. 44, no. 7, pp. 1259-1273, 2002.

[8] B. A. T. Petersson, "Structural acoustic power transmission by point moment and force excitation, part II: plate-like structures," Journal of Sound and Vibration, vol. 160, no. 1, pp. 67-91, 1993.

[9] R. Gunda, S. M. Vijayakar, and R. Singh, "Method of images for the harmonic response of beams and rectangular plates," Journal of Sound and Vibration, vol. 185, no. 5, pp. 791-808, 1995.

[10] J. Liang and B. A. T. Petersson, "Estimation of vibration distribution for finite structures," Journal of Sound and Vibration, vol. 238, no. 2, pp. 271-293, 2000.

[11] S. Elias and V. Matsagar, "Research developments in vibration control of structures using passive tuned mass dampers," Annual Reviews in Control, vol. 44, pp. 129-156, 2017.

[12] A. Palazzolo, Vibration Theory \& Applications with Finite Elements \& Active Vibration Control, John Wiley \& Sons, Hoboken, NJ, USA, 2016.

[13] A. Preumont, Vibration Control of Active Structures: An Introduction, Springer, Berlin, Germany, 2011.

[14] C. Du and L. Xie, Modeling and Control of Vibration in Mechanical Systems, Taylor \& Francis Group, LLC, Boca Raton, FL, USA, 2010.

[15] C. Wei and X. Jing, "A comprehensive review on vibration energy harvesting: modelling and realization," Renewable and Sustainable Energy Reviews, vol. 74, pp. 1-18, 2017.

[16] T. Yildirim, M. H. Ghayesh, W. Li, and G. Alici, "A review on performance enhancement techniques for ambient vibration energy harvesters," Renewable and Sustainable Energy Reviews, vol. 71, pp. 435-449, 2017.

[17] N. Tran, M. H. Ghayesh, and M. Arjomandi, "Ambient vibration energy harvesters: a review on nonlinear techniques for performance enhancement," International Journal of Engineering Science, vol. 127, pp. 162-185, 2018.

[18] H. Xiao, X. Wang, and S. John, "A multi-degree of freedom piezoelectric vibration energy harvester with piezoelectric elements inserted between two nearby oscillators," Mechanical Systems and Signal Processing, vol. 68-69, pp. 138-154, 2016.

[19] Z.-c. Qiu, X.-m. Zhang, H.-x. Wu, and H.-h. Zhang, "Optimal placement and active vibration control for piezoelectric smart flexible cantilever plate," Journal of Sound and Vibration, vol. 301, no. 3-5, pp. 521-543, 2007.

[20] C. M. A. Vasques and J. Dias Rodrigues, "Active vibration control of smart piezoelectric beams: comparison of classical and optimal feedback control strategies," Computers \& Structures, vol. 84, no. 22-23, pp. 1402-1414, 2006.

[21] E. Omidi and S. N. Mahmoodi, "Active vibration control of structures using a leader-follower-based consensus design," Journal of Vibration and Control, vol. 24, no. 1, pp. 60-72, 2018.

[22] E. Omidi and N. Mahmoodi, "Hybrid positive feedback control for active vibration attenuation of flexible structures," IEEE/ASME Transactions on Mechatronics, vol. 20, no. 4, pp. 1790-1797, 2015. 